Received: 2 June 2017

Accepted: 16 February 2018

Published online: 01 March 2018
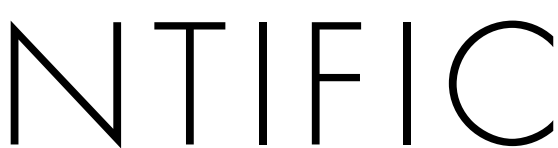

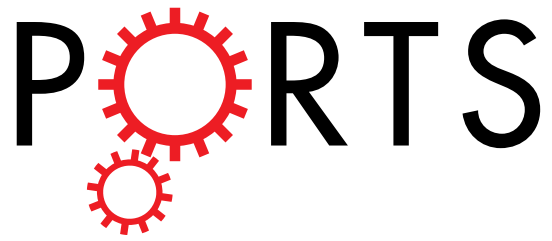

\title{
OPEN
}

\section{Transcriptional activation of} zebrafish fads2 promoter and its transient transgene expression in yolk syncytial layer of zebrafish embryos

\begin{abstract}
Shu-Shen Tay ${ }^{1}$, Meng-Kiat Kuah ${ }^{2}$ \& Alexander Chong Shu-Chien $\mathbb{1}^{1,2}$
The front-end desaturases (Fads) are rate-limiting enzymes responsible for production of long-chain polyunsaturated fatty acids (LC-PUFA). The full spectrum of the transcriptional regulation of $f a d s$ is still incomplete, as cloning of fads promoter is limited to a few species. Here, we described the cloning and characterisation of the zebrafish fads 2 promoter. Using $5^{\prime}$-deletion and mutation analysis on this promoter, we identified a specific region containing the sterol regulatory element (SRE) which is responsible for the activation of the fads 2 promoter. In tandem, two conserved CCAAT boxes were also present adjacent to the SRE and mutation of either of these binding sites attenuates the transcriptional activation of the fads 2 promoter. An in vivo analysis employing GFP reporter gene in transiently transfected zebrafish embryos showed that this 1754 bp upstream region of the fads2 gene specifically directs GFP expression in the yolk syncytial layer (YSL) region. This indicates a role for LC-PUFA in the transport of yolk lipids through this tissue layer. In conclusion, besides identifying novel core elements for transcriptional activation in zebrafish fads 2 promoter, we also reveal a potential role for fads 2 or LCPUFA in YSL during development.
\end{abstract}

In eukaryotic cells, the front-end desaturases (Fads) catalyse the introduction of a double bond at fixed number of carbons from the carboxyl group. A typical Fads possesses characteristic features such as three histidine-rich boxes, transmembrane regions, and an $\mathrm{N}$-terminal cytochrome $\mathrm{b}_{5}$ domain containing the heme-binding motif HPGG. Long-chain polyunsaturated fatty acids (LC-PUFA) such as arachidonic acid (ARA, 20:4n-6), eicosapentaenoic acid (EPA, 20:5n-3) and docosahexaenoic acid (DHA, 22:6n-3) are generated from shorter polyunsaturated fatty acids (PUFA) through the actions of Fads and elongases (Elovl). LC-PUFA are crucial for maintenance of cellular membrane integrity, as precursors for eicosanoids, regulation of gene expression and signal transduction pathways. In aquaculture, the interest to decipher the activities of the LC-PUFA biosynthesis enzymes and transcription factors involve in LC-PUFA biosynthesis is driven by the pressing need to improve performance of vegetable oils in aquafeeds ${ }^{1}$. These oils are lacking in LC-PUFA but rich in $\mathrm{C}_{18}$ PUFA. Understandably, a greater understanding of distinctive bioconversion capacities of $\mathrm{C}_{18}$ PUFA to LC-PUFA in different farmed species could theoretically improve the strategy of employing vegetable oil as feeds ${ }^{2}$.

Among the vertebrates, numerous studies have reported the molecular cloning and characterisation of Fads from different fish species ${ }^{1}$. Fads from mammals are principally mono-functional, with $\Delta 5$ and $\Delta 6$ desaturations being carried out by two separate genes, Fads 1 and Fads2, respectively ${ }^{3}$. In fish, fads 1 have so far been isolated from only one species, a basal gnathostome, leading to the hypothesis that a complete loss of fads 1 in teleosts have occurred following gnathostome radiation ${ }^{4}$. Isolation and characterisation of teleost fads 2 to date have reported a broad range of substrate specificities, with $\Delta 4, \Delta 5, \Delta 6$ and $\Delta 8$ desaturation capacities reported. In addition, both mono-functional and bi-functional fads 2 have been isolated from a myriad of teleost species ${ }^{5-7}$. As compared to freshwater species, marine teleosts seemed to display a lesser capacity for bioconversion of $\mathrm{C}_{18}$ PUFA to LC-PUFA,

${ }^{1}$ School of Biological Sciences, Universiti Sains Malaysia, 11800, Minden, Penang, Malaysia. ${ }^{2}$ Centre for Chemical Biology, Universiti Sains Malaysia, Sains@USM, Block B No. 10, Persiaran Bukit Jambul, 11900, Bayan Lepas, Penang, Malaysia. Correspondence and requests for materials should be addressed to A.C.S.-C. (email: alex@usm.my) 
due to the loss of crucial $\mathrm{fads}$ from its genome or low activities of $f a d s 2$ or $\mathrm{elovl}$. This was postulated to be a result of richer LC-PUFA content in the marine environment, causing diminished capacity for LC-PUFA biosynthesis. Subsequent works however advocated the influence of feeding niche on LC-PUFA biosynthesis, when a catalogue of fads 2 and elovl were isolated from marine fish species which possess a diet with limited DHA intake ${ }^{6,9}$.

Despite the multiple interests in molecular characterisation of $f a d s$ in teleost, there is a paucity of understanding on its regulation at the transcriptional level. It is conceivable that differences in regulatory activities by dissimilar transcription factors responding to different cues including nutritional status is responsible for the different LC-PUFA biosynthesis capacity in various fish species. These transcription factors themselves are subjected to regulation by the nutritional status of the animal. Therefore, efficient use of vegetable oils in aquafeeds will benefit from the ability to decipher the link between the overall lipid metabolic pathways modification caused by the diet and the subsequent influence on lipid homeostasis genes at the transcriptional level. To date, promoter sequences from teleost fads 2 have been isolated from Atlantic salmon ${ }^{10}$, Atlantic $\operatorname{cod}^{10}$, rainbow trout, European sea bass ${ }^{11}$, Japanese seabass ${ }^{12}$, large yellow croaker ${ }^{13}$ and rabbit fish ${ }^{14}$. Firstly, these works collectively showed the presence of binding sites for transcription factors known to play a role in mammalian cholesterol and lipogenic synthesis pathways such as sterol regulatory element-binding protein (Srebp), nuclear factor Y (NF-Y) and ubiquitous transcription factor Sp1 in the fads 2 promoter ${ }^{10-14}$. Secondly, these studies suggested an interplay between different transcription factors to mediate the regulation of $f a d s 2$ transcription.

The zebrafish has gained reputation as a useful model in understanding the role of lipids and fatty acids during development ${ }^{15,16}$. Largely driven by the existence of multiple homologous genes involved in conserved lipid and lipoprotein metabolism network, zebrafish have been used to gain insight on lipid adsorption, atherosclerosis, fatty liver disease and obesity ${ }^{15,17}$. Zebrafish tissues are reported to contain significantly higher concentrations of PUFA than equivalent mammalian tissues ${ }^{18,19}$. Molecular cloning, functional characterisation and expression profile of fads 2 and several elovl genes during embryogenesis have been reported in zebrafish ${ }^{5,20-23}$. However, our knowledge on the function of fads $2 /$ elovl and LC-PUFA during development is still fragmentary. Given how important transcriptional regulation in influencing the activities of fads 2 may be and how little is known about the regulators and cellular conditions for expression, it is opportune to use zebrafish as a model to fill these gaps.

In this present study, we described the cloning and characterisation of the zebrafish fads 2 promoter. This includes identification and functional characterisation of pivotal regulatory elements responsible for driving transcriptional activities using luciferase reporter gene in a zebrafish cell line. In addition, we also carried out an in vivo characterisation of the zebrafish fads 2 promoter using a transient approach to track its expression in developing zebrafish embryos.

\section{Results}

Cloning and characterisation of the zebrafish fads2 promoter. We isolated a putative promoter region of $1754 \mathrm{bp}$, comprising $19 \mathrm{bp}$ of the $5^{\prime}$-UTR of zebrafish $\Delta 5 / \Delta 6 \mathrm{fads} 2^{5}$ and $1735 \mathrm{bp}$ of the upstream region. For positioning purpose, +1 was given to the putative transcription start site (TSS) derived from comparison of the $5^{\prime}$-end of full-length cDNA (GenBank accession number: NM_131645.2) and DNA sequences of the fads2 gene. Within this fads 2 promoter region, a classic transcription initiation element, the TATA box was identified at the location of $-26 \mathrm{bp}$ (Supplementary data 1$)$.

Zebrafish Srebp upregulates fads2 promoter activity. Co-transfection of ZFL cells with the fads21735 promoter-luciferase reporter plasmid and expression plasmid containing either the nuclear form of zebrafish Srebp1 (nSrebp1) or Srebp2 (nSrebp2) proteins, respectively, resulted in significantly higher reporter activities than cells transfected with empty pcDNA3.1 expression plasmid (Fig. 1). Among the two isoforms, the zebrafish $\mathrm{nSrebp} 2$ protein resulted in higher luciferase reading. This signified the importance of Srebp proteins in driving the expression of zebrafish fads2, and subsequent luciferase reporter transactivation assays were performed with the overexpression of zebrafish nSrebp2 protein.

Identification of cis-regulatory elements within the $-214 /-67$ bp region of zebrafish fads2 promoter. To identify the presence of any potential cis-regulatory element, the nucleotide sequence within the $-214 /-67 \mathrm{bp}$ region of zebrafish $\mathrm{fads} 2$ promoter was aligned and compared to corresponding sequences from rainbow trout ${ }^{13}$ (GenBank accession number: KT781408.1), Atlantic salmon ${ }^{10}$ (GenBank accession number: AY736067.2), European seabass ${ }^{11}$ (GenBank accession number: FP671139.1), large yellow croaker ${ }^{13}$ (GenBank accession number: KT781409.1), Japanese seabass ${ }^{12}$ (GenBank accession number: KT781410.1), Atlantic cod ${ }^{10}$ (GenBank accession number: FJ859898.1), human ${ }^{24}$ (GenBank accession number: AP002380.3) and mouse (GenBank accession number: AC135670.3). The alignment disclosed the presence of several conserved putative transcription factor binding sites, namely two inverted CCAAT boxes, an inverted ubiquitous transcription factor SP1 site and a sterol regulatory element (SRE) within the cloned promoter region (Fig. 2). The result also showed that the CCAAT boxes were highly conserved across the mammalian and fish species while the SRE (CTCGAATGATC) found in the zebrafish fads2 promoter was identical to those in all the teleost species but differed from the mammalian SRE (CTCTGCTGATC) by three consecutive nucleotides. The SP1 binding site is less conserved amongst the mammalian and fish species, with two SP1 binding site in the mammalian Fads2 promoters, while teleost fads 2 promoters typically contain either a single SP1 site or none.

$-161 /-67$ bp region is critical for zebrafish fads2 promoter activation. We sought to determine a minimal fads 2 promoter fragment capable of activating transcription by fusing a series of $5^{\prime}$-deleted promoter fragments to the luciferase reporter gene. Upon normalisation to values obtained with a promoter-less pGL3.1 plasmid, the intact fads2 promoter $(-1735 /+19 \mathrm{bp})$ drove a luciferase activity of approximately 200 -fold (Fig. 3). Deletion of promoter up to $161 \mathrm{bp}$ upstream of the TSS did not greatly diminish transcription activities, notwithstanding some reduction in 


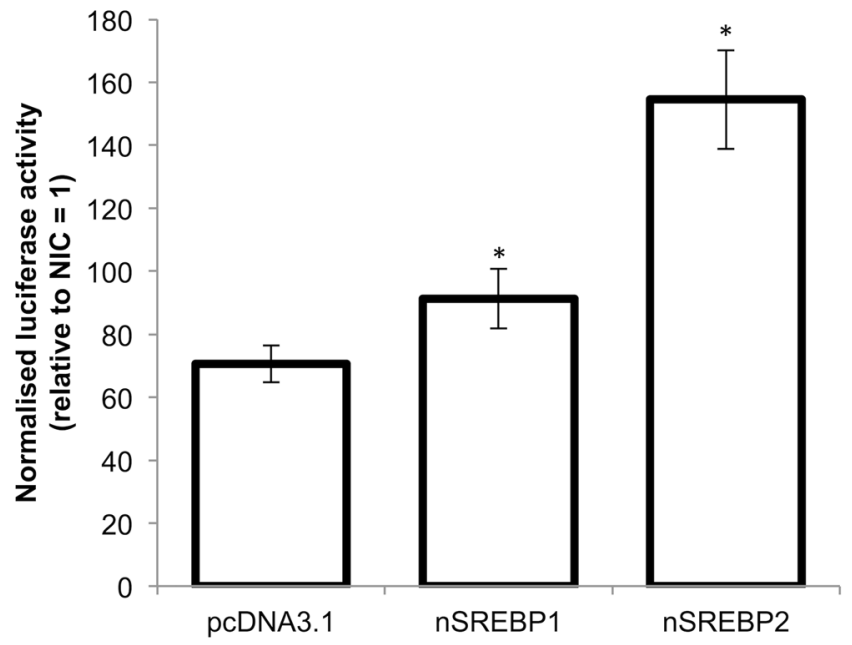

Figure 1. Effects of nSrebp proteins on the zebrafish fads2 promoter activation. ZFL cells were co-transfected with nSrebp1 or nSrebp2 expression plasmid (empty pcDNA3.1 expression plasmid as control), and pGL3 promoter-luciferase reporter plasmid fused with the zebrafish fads2 desaturase promoter $(-1735 /+19 \mathrm{bp})$. Results represent normalised luciferase activity (to Renilla luciferase activity) of fads 2 promoter in the ZFL cells relative to empty pGL3-Basic plasmid. Values are means $\pm \mathrm{SD}(\mathrm{n}=3) ; P<0.05$ by one-way ANOVA.

luciferase readings as compared to the longer fragments. However, further deletion of the sequence within $-161 /-67$ bp almost completely abolished the promoter activity, which indicated a mandatory site within the region of -161 to $-67 \mathrm{bp}$ for activation of the fads 2 activities. This region contains both CCAAT boxes and the SRE (Supplementary data 2). This also means the removal of the SP1 was not detrimental to the activation of zebrafish fads 2 promoter.

CCAAT boxes and SRE mediates fads2 promoter activity. We introduced single or multiple site mutations for the SRE, proximal CCAAT box (CATp) and distal CCAAT box (CATd) to the fads2-244 promoter-luciferase reporter plasmid, followed by the dual luciferase reporter assay in ZFL (Fig. 4). Firstly, results showed that both CATp and CATd could drive expression of promoter, as seen in the fragment with mutated SRE, although the expression was lower than the fragment with all three binding sites intact. In comparison, disrupting both proximal and distal CCAAT bindings sites resulted in total abolishment of luciferase expression, an observation which was recapitulated when all three binding sites were mutated. This implies an obligation for both CCAAT and SRE to optimally drive zebrafish $f a d s 2$, although the former was still able to activate a reduced measure of transcriptional activities. When SRE was present, CATp seemed to play a more prominent role as compared to CATd in driving promoter activation. A similar pattern was also observed when SRE was disrupted.

In vitro binding of ZFL nuclear proteins at the SRE and CCAAT regions in the zebrafish fads2 promoter. Based on the observations from the mutagenesis experiments, we ran EMSA to assess the binding capacities of transcription factors in ZFL nuclear extract to the fads2 promoter cis-regulatory elements. Results showed the formation of DNA-protein complex when ZFL cells nuclear extract was incubated with wild type oligonucleotide probe containing CATp binding site. There was no complex formed when the probe was incubated without the nuclear extract or when a probe with mutated CATp sequence was used with the nuclear extract. Similarly, the use of non-labelled wild type oligonucleotides in excess amount completely outnumbered the formation of labelled DNA-protein complex. A comparable pattern was shown with CATd sequence (Fig. 5).

Unlike the CATp and CATd probes, DNA-protein complex was not observed when nuclear proteins were added into the sample containing oligonucleotides with only SRE site (result not shown). Taking this and the results from mutagenesis experiment into consideration, we used a longer oligonucleotide consisting of both SRE and the adjacent CATp. Results showed that binding of transcription factors and DNA occurred with the simultaneous presence of both the SRE and CATp binding sites (Fig. 6). Mutation on either site prevented the formation of any DNA-protein complex. When the wild type labelled probe was incubated with excessive non-labelled probe containing mutated SRE and intact CATp, a decrease in DNA-complex staining was observed due to competition for binding. This result encored the observation in cell-based luciferase assay which showed the requirement of CATp for activation of reporter gene expression. In contrast, when wild type labelled probe was incubated with excessive non-labelled probe with intact SRE and mutated CATp binding site, the formation of DNA-complex occured. Therefore, in the absence of any conjugated protein binding to CATp, the binding of Srebp to the SRE was perturbed. Taken together, the results from mutagenesis and EMSA suggested a functional role for CCAAT box in the Srebp dependent transcriptional activation of zebrafish fads 2 promoter.

Transient expression of fads2 promoter (-1735/+19 bp) in developing zebrafish embryos. We observed GFP signal in the yolk syncytial layer (YSL) of embryos beginning at $26 \mathrm{hpf}$ for both the pZs $-244 \mathrm{bp}$ and pZs-1735 bp constructs, respectively. As embryos developed, higher percentage of embryos with YSL-GFP 
SP1

SP1

Danio rerio

oncorhynchus mykiss

Salmo salar

Dicentrarchus labrax

Larimichthys crocea

Lateolabrax japonycus

Gadus morhua

Mus musculus

Homo sapiens

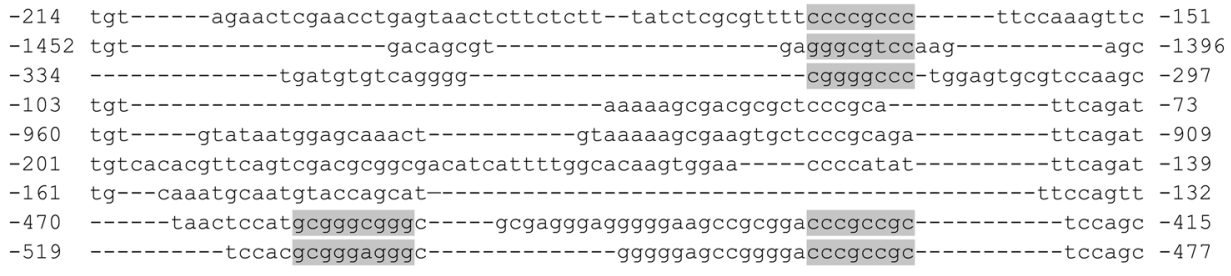

CCAAT box

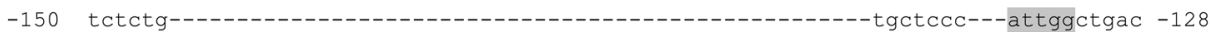

-1395 gtcctgattggttgaaataggcaagcctttgacagtcctgaaggccaattcggataggctgttgtgaaccaat-tgca -1319

-296 gttctgattggttgaaatatgtggatgattgacagacctgaag-------------ggcttttttgaaccaat-tgca -233

-72 gagctg---

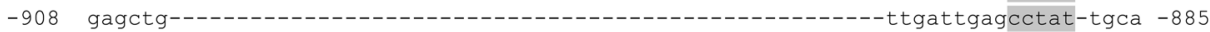

-138 aagctg-------------------------------------------------tttattgagcctat-tgca -115

-131 caactgtactgctgaaacgcacgccacacgcacgccacatttgccagcggtcgg--ggatacgcgcggattgg----- -61

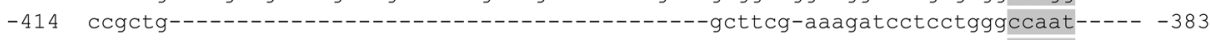

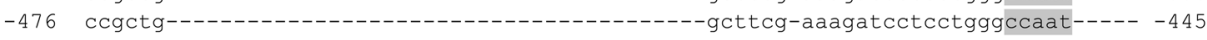

$\star * *$

CCAAT box

SRE

-127 agtccg-gagactctctca--gagacgc-------gcgccgattggctgctggag---ctcgaatgatctgtt -67

-1318 gatatgccag-cggtctagt-gaataac---------cccattgg--acgagaga--ctcgaatgatcggca -1261

-232 gatatgccag-gggtctatt-gaataac---------cccattgg--actagagaccetcgaatgatctgct -173

-48 catcagccag-cggtctag--gatatac----tgtacgecgattgg--cccagaaaccetcgaatgatcgget +16

-884 catcagccag-tggtccag--catgtac----tgtactccgattgg--ctcagaaaccetcgaatgatcggca -821

-114 catgagccag-tggtccag--gatatacctactgtgcgccgattgg--cccagaaagcctcgaatgatcagct -47

-60 -----------tccgg--gatacgc--------gcggattgg--cccaccatccetcgaatgatcgctc -15

-382 ----ggcagg-----cggggcgacgagcc---------ctgattgg-tgcaggcg---ctctgctgatcactg -332

-444 ----ggcagg-----cggggcgacgcgac--------cggattgg-tgcaggcg---ctctgctgatcgctg -394

Figure 2. Conserved cis-regulatory elements within the core promoter regions of the fads 2 genes from zebrafish and other vertebrate counterparts. Sequence alignment was performed with web-based program, MAFFT (version 7). Numbers indicating the sequence positions are relative to TSS $(+1)$, based on the cDNA sequence information deposited in the GenBank (Danio rerio: CU694371.13; Oncorhynchus mykiss: KT781408.1; Salmo salar: AY736067.2; Dicentrarchus labrax: FP671139.1; Larimichthys crocea: KT781409.1; Lateolabrax japonycus: KT781410.1; Gadus morhua: FJ859898.1; Mus musculus: AC135670.3 and Homo sapiens: AP002380.3).

Conserved regions for the SP1 binding site, CCAAT boxes and SRE consensus sequence are highlighted in grey, while identical nucleotides are indicated with asterisks.

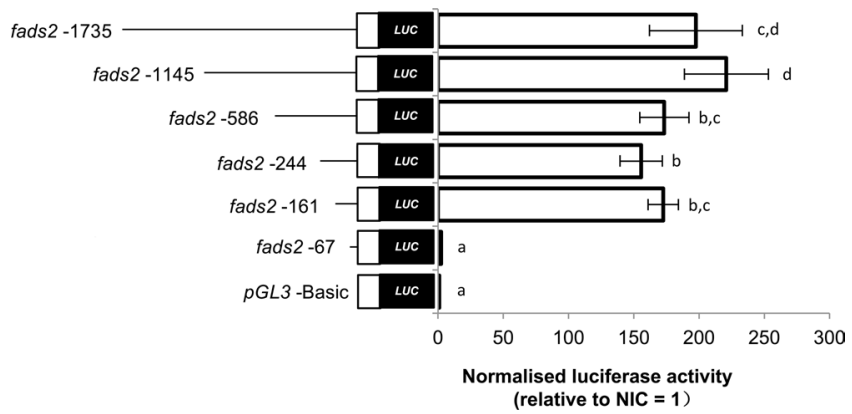

Figure 3. $5^{\prime}$-deletion analysis of the zebrafish fads 2 promoter. ZFL cells were transiently co-transfected with the fads $25^{\prime}$-deletion promoter-luciferase reporter plasmids, nSrebp2 expression plasmid together with Renilla luciferase reference plasmid pRL-SV40. $5^{\prime}$-deletion plasmids were named according to respective position in relation to TSS $(+1)$ and are represented by horizontal line on the left. Non-coding exon is indicated with open boxes. Luciferase activity of fads 2 promoter in ZFL cells is expressed as normalised luciferase activity (to Renilla luciferase activity) relative to empty pGL3-Basic plasmid. Values are means \pm S.D. $(n=3)$ (right panel). Groups indicated with different letters are significantly different (Tukey's test; $P<0.05$ ).

expression were observed, along with increase in GFP signal intensity (Fig. 7). At 120 hpf, more than 95\% of injected embryos showed GFP expression at the YSL (Table 1). Therefore, a fads 2 promoter fragment containing response elements such as SRE, CATp and CATd proven to be essential for activation of expression is now shown to drive a spatio-temporal expression of fads 2 in the zebrafish YSL. 


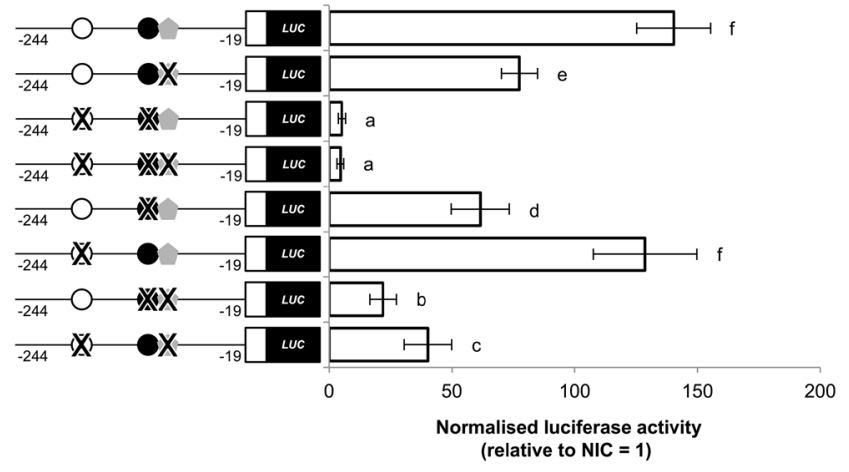

Figure 4. Mutated CCAAT box and SRE reduce transcriptional activity of the zebrafish fads 2 promoter. Single or multiple site mutations $(\mathbf{X})$ of SRE $(\triangle)$, CATp $(\mathbf{O})$ and CATd $(O)$ were introduced into the fads $2-244$ plasmid. ZFL cells were transiently co-transfected with various mutated fads2-244 plasmids, nSrebp2 expression plasmid, together with Renilla luciferase reference plasmid pRL-SV40. Luciferase coding region is indicated by shaded box and activity of the fads 2 promoter in ZFL cells is expressed as normalised luciferase activity (to Renilla luciferase activity) relative to the empty pGL3-Basic plasmid. Values are means \pm SD of three independent experiments $(\mathrm{n}=3)$ (right panel). Groups indicated with different letters are significantly different (Tukey's test; $P<0.05)$.

Oligonucleotide:
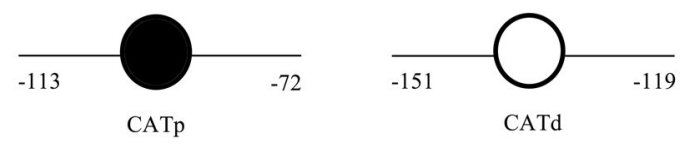

\begin{tabular}{lcccc|cccc}
\hline \multirow{2}{*}{ Lane } & \multicolumn{4}{c|}{ CATp } & \multicolumn{4}{c}{ CATd } \\
\cline { 2 - 8 } & $\mathbf{1}$ & $\mathbf{2}$ & $\mathbf{3}$ & $\mathbf{4}$ & $\mathbf{1}$ & $\mathbf{2}$ & $\mathbf{3}$ & $\mathbf{4}$ \\
\hline wt & + & + & - & + & + & + & - & + \\
mut & - & - & + & - & - & - & + & - \\
wt competitor & - & - & - & + & - & - & - & + \\
Nuclear extract & - & + & + & + & - & + & + & +
\end{tabular}

DNA-protein complex

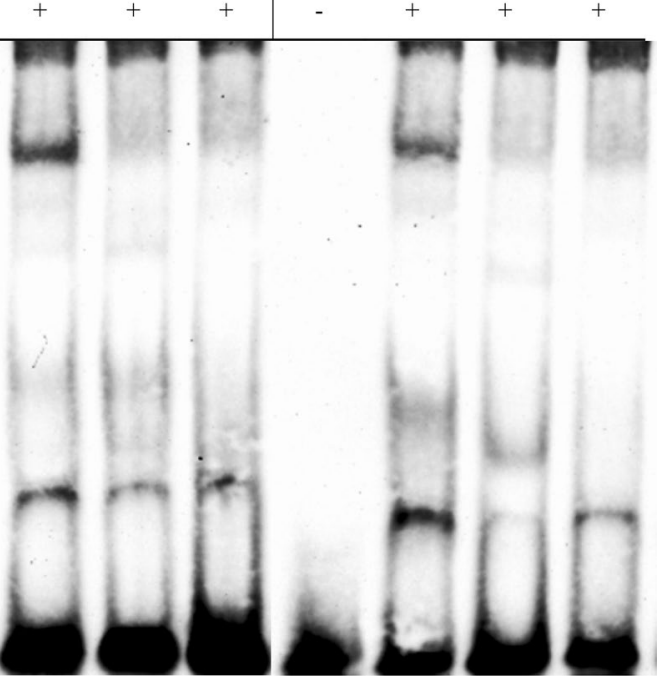

Figure 5. Binding of ZFL nuclear proteins to the proximal CCAAT box (CATp) and distal CCAAT box (CATd) on the zebrafish $\mathrm{fads} 2$ promoter. EMSA was performed with biotin-labelled oligonucleotides containing CATp or CATd box and nuclear extract of ZFL cells. Specific binding of DNA-protein complex was validated with site-mutated oligonucleotides (Lane 3) and 100-fold molar excess of non-labelled competition oligonucleotides (Lane 4).

\section{Discussion}

The zebrafish fads 2 was first cloned and shown to have the capacity for in vitro $\Delta 5 / \Delta 6$ desaturation ${ }^{5}$. The transcripts were reported to express in several tissues known for biosynthesis of LC-PUFA during embryonic development $^{21}$. The mRNA transcript of zebrafish fads 2 is also seemingly regulated by dietary PUFA intake ${ }^{25}$. Despite these studies, investigation on the regulation of fads 2 expression at transcription level is lacking. Here, we cloned 
Oligonucleotide:

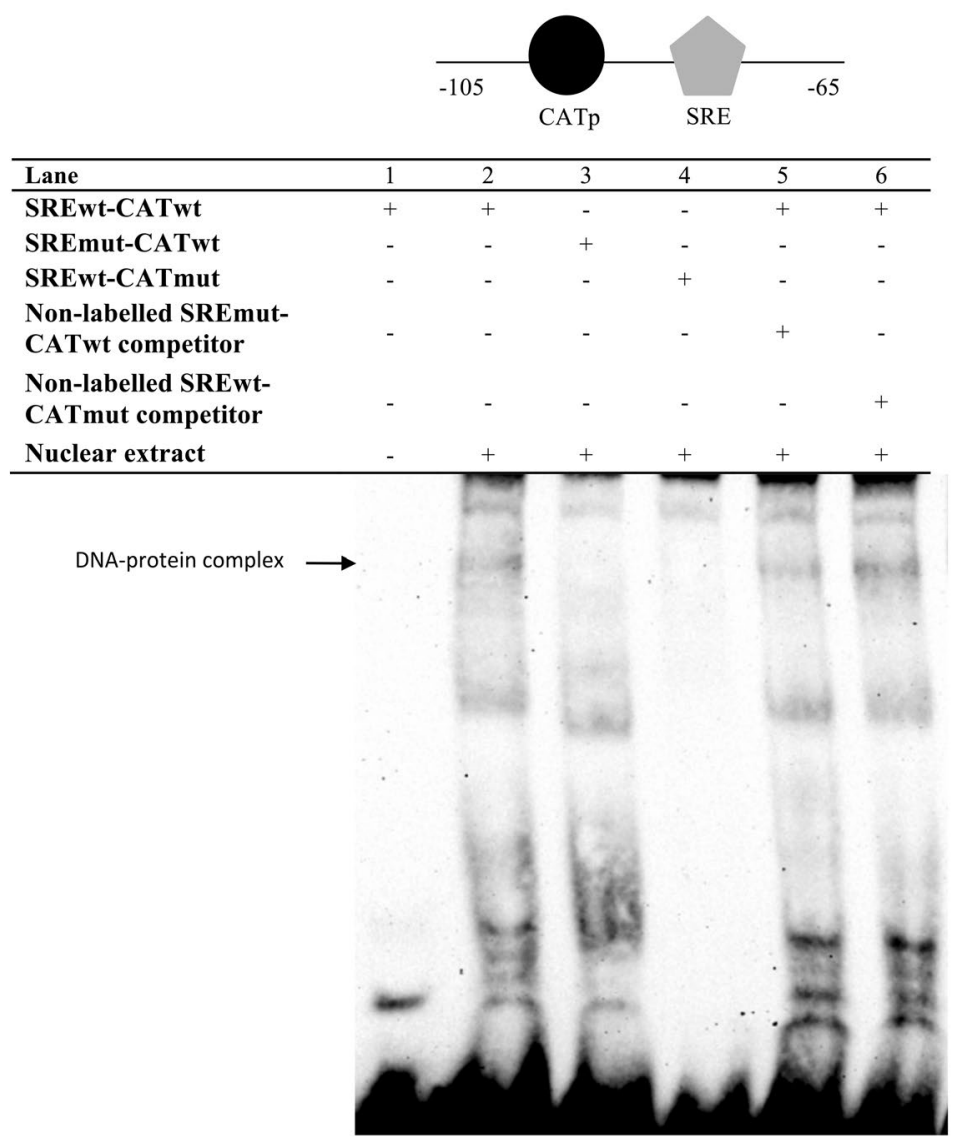

Figure 6. Binding of ZFL nuclear proteins to the SRE on the zebrafish fads2 promoter. Biotin-labelled probes containing SRE and proximal CCAAT box (CATp) were incubated with the nuclear extract of ZFL cells. Specific binding of DNA-protein complex was validated with site-mutated oligonucleotides (Lanes 3 and 4) and 100-fold molar excess of non-labelled mutated competition oligonucleotides (Lane 5 and 6).

a 1754 bp promoter region of the zebrafish $f a d s 2$ gene to determine response elements critical for control of $f a d s 2$ expression. We showed here that like all known teleost fads 2 promoters, zebrafish fads 2 promoter harbours SRE and CCAAT binding elements ${ }^{10-13}$. Zheng et al. ${ }^{10}$ also showed a high degree of sequence conservation between mammalian, amphibian and teleost species, which indicates conserved mechanism of fads 2 role. As with salmon ${ }^{10}$ and trout ${ }^{13}$, putative SP1 binding site was also identified in this region of zebrafish fads 2 promoter. In contrast, all sequenced promoters of $f a d s 2$ from marine teleost species so far do not contain SP1 element, which was postulated as a reason for lower fads 2 expression in marine fish species ${ }^{11-13}$.

Results from the promoter deletion experiment demarcated regions responsible for transcriptional activation of the zebrafish fads2. One such region is the cis-element SRE, the binding site for Srebp transcription factors. Using mutagenesis approach, we further corroborate the obligation of the SRE region in activating transcription of zebrafish fads2. We also showed that this sequence forms a DNA-protein complex with nuclear extract of ZFL cells. In human, an E-box like SRE region, as opposed to the classical SRE region, was crucial for both activation and suppression of the FADS2 gene ${ }^{24}$. Srebps are prominent regulators of lipid, fatty acid and cholesterol biosynthesis, and were first identified as nuclear proteins possessing binding abilities to SRE of low density lipoprotein receptor $^{26}$. Like mammals, fish have two distinct homologues of mammalian Srebp1 and Srebp $2^{27}$. In zebrafish, activation of hepatic Srebp induce the expression of both lipid and cholesterol biosynthesis genes ${ }^{28}$. Our results also showed that in ZFL cells, Srebp2 activated a higher level of fads 2 transcription as compared to Srebp1. This is reminiscent of the findings in Atlantic salmon, where Srebp2 promoted higher fads 2 activities as compare to Srebp $1^{29}$. The importance of Srebp in mediating teleost fads2 expression has been shown through the use of cell-based luciferase assays involving Srebp expression plasmids or mutation of the promoter's SRE response element ${ }^{13,14,29}$. In terms of tissue localisation, there was a parallel pattern between the distribution of srebp and fads2 mRNA expression ${ }^{27,30}$. The role of Srebp as a sensory mediator between dietary LC-PUFA intake and LC-PUFA biosynthesis was also observed in several fish species wherein limited dietary LC-PUFA intake led to increased Srebp level, subsequently resulting in higher fads 2 mRNA expression ${ }^{13,29-32}$. In rat and human, dietary PUFA inhibits the rate of Fads 2 transcription through Srebp, probably by lessening the production of mature Srebp or accelerating the decay of Srebp mRNA ${ }^{24,33}$. Besides FADS2, Srebp have also been shown to regulate ELOVL ${ }^{24,34}$. 


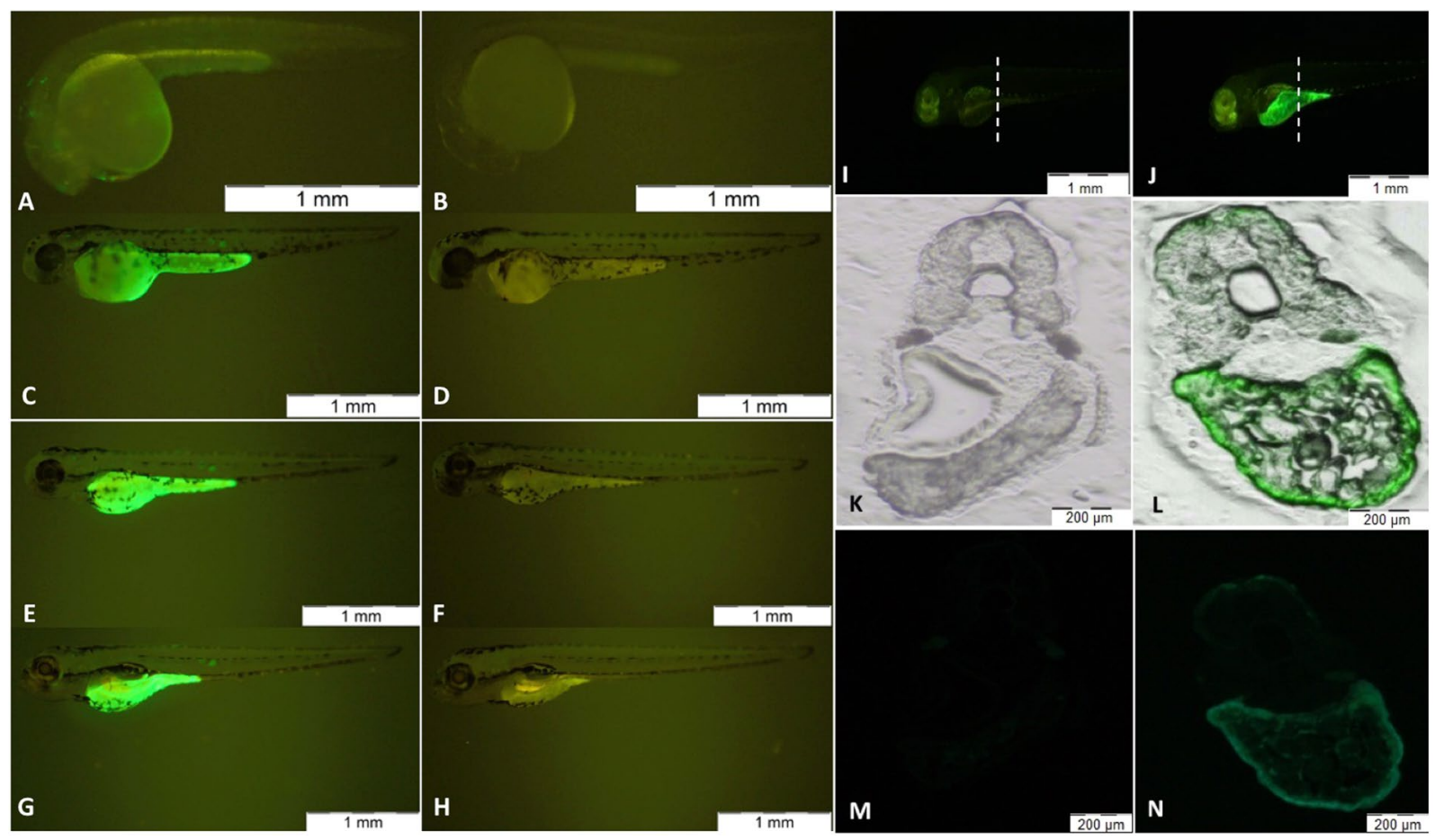

Figure 7. Transient GFP expression of linearised fads 2 promoter $(-1735 /+19 \mathrm{bp})$ localised in yolk syncytial later (YSL) of zebrafish embryos. Lateral view of 26, 48, 72 and $96 \mathrm{hpf}$ of transient transgene embryo injected with linearised fads 2 promoter $(-1735 /+19 \mathrm{bp})$ showing increased expression in YSL $(\mathbf{A}, \mathbf{C}, \mathbf{E}, \mathbf{G})$ and their corresponding control (B,D,F,H). Cross-sectional view of a $120 \mathrm{hpf}$ control embryo (K-N) and a $120 \mathrm{hpf}$ transient transgenic embryo $(\mathbf{I}, \mathbf{J})$ injected with linearised fads2 promoter $(-1735 /+19 \mathrm{bp})$ showing expression at YSL.

\begin{tabular}{|l|l|}
\hline Description & Embryo number (\%) \\
\hline Injected & 72 \\
\hline Survival & $63(87.5 \%)$ \\
\hline Dead/Abnormal & $9(12.5 \%)$ \\
\hline GFP expressing embryos & $58(92.1 \%)$ \\
\hline Localisation of GFP & $57(98.3 \%)$ \\
\hline YSL & $1(1.7 \%)$ \\
\hline Non-specific (notochord) &
\end{tabular}

Table 1. Transient expression of the linearised fads 2 promoter $(-1735 /+19 \mathrm{bp})$ in zebrafish embryos. Figures are cumulative embryos scored from three independent microinjections at $120 \mathrm{hpf}$.

Through a combination of deletion and mutagenesis, we demonstrate the importance of two CCAAT motifs in the core promoter region of zebrafish fads 2 for transcriptional activation. The CCAAT box, named after the five nucleotides customarily found in the binding sites, is one of the most ubiquitous binding element in eukaryotic promoters, with several corresponding binding proteins already isolated and characterised ${ }^{35}$. The NF-Y is the major protein recognizing the CCAAT box, and is obligatory for the transcription of a considerable number of genes with ubiquitous or tissues-specific expression pattern ${ }^{35}$. Functional CCAAT motifs recognised by NF-Y are among the most frequent boxes found in promoters ${ }^{36}$. Mutation of putative NF-Y binding sites in fads2 promoter of two marine teleost species also resulted in weaker transcriptional activities ${ }^{10,14}$.

Our present finding suggests that the putative NF-Y binding sites are required for formation of complex between Srebp and SRE, which then activates the transcription of reporter gene. This result resonated with earlier findings wherein Srebp was incapable of driving transcription of target genes if adjacent NF-Y binding sites were mutated $^{37-39}$. By themselves, Srebp proteins are inherently weak transcriptional activators, which underlines the need for Srebp proteins to interact with additional transcriptional factors to regulate the expression of lipogenic genes ${ }^{38,40,41}$. Studies on promoters of cholesterol and fatty acid metabolism genes consistently highlight the role of NF-Y as a pivotal partner in Srebp-mediated regulation of transcriptional activities ${ }^{36,38,41-43}$. Elsewhere, studies on murine stearoyl-CoA desaturase $(S c d)$ and human FADS2 promoters both showed a requirement for putative NF-Y binding sites located adjacent to SRE for transcriptional activation ${ }^{24,39}$. Promoters of teleost and amphibian fads genes also contain a conserved region bearing NF-Y and Srebp binding sites at similar positions relative to the transcriptional start site (TSS) ${ }^{10}$. It is speculated that direct interaction of Srebp and NF-Y, together with the 
binding of both these transcription factors to neighbouring sites on the DNA lend stability to the DNA-protein complex ${ }^{39}$. In addition, our results showed that removal of the putative SP1 binding region did not supress the luciferase expression. The interaction between Srebp and SP1 in the regulation of Fads have not been demonstrated this far ${ }^{24,33}$, although a synergistic type of activation between these two transcriptional factors have been elucidated in other lipid metabolism genes ${ }^{44}$. A role for SP1 in Srebp-mediated activation of Fads2 however cannot be ruled out as different isoforms of Srebp or nutritional conditions may utilise different co-regulatory factors to activate expression of Fads, as shown in promoters of fatty acid synthase ${ }^{45}$.

Fatty acids from yolk storage are critical for energy and structural components for both fish embryo and larval stages ${ }^{46}$. Together with the head region, yolk sac has been reported to contain majority of the lipids during zebrafish embryonic stages ${ }^{19}$. While saturated and monounsaturated fatty acids (SFA, MUFA) are consumed for energy, LC-PUFA are essential for cellular membrane integrity, regulation of gene transcription, regulation of signalling pathways and production of eicosanoids. Previously, the yolk was regarded as a non-metabolic active site for nutrient reserve, where molecules such as lipids are stored within yolk cells and transported when needed ${ }^{46,47}$. However, emerging findings employing zebrafish as the model organism, are recognising the yolk as site for active lipid metabolism preceding transportation ${ }^{16}$. Using a transient transgenic approach in zebrafish embryos, we showed that the $-1735 /+19 \mathrm{bp}$ region of the fads 2 promoter directed a specific expression in the YSL beginning at $26 \mathrm{hpf}$ embryos. In support of this, fads2 and elovl transcripts were reported to be expressed in YSL at the 24 hpf stage, alongside brain tissues ${ }^{18,21}$. During early embryonic development, some of the blastomeres situated at surface of the yolk collapse and fuse with the yolk cells, leading to the formation of YSL ${ }^{48,49}$. It then undergoes a series of changes in terms of thickness and nuclei distribution, before going into the process of degradation upon the exhaustion of yolk supplies ${ }^{50}$. YSL governs major roles during development, including yolk metabolism, epiboly, specification of germ layers, organogenesis of endoderm organs and innate immunity ${ }^{50-52}$. A major role of YSL in teleost development is on the transport of nutrients from yolk to embryonic cells and tissues. Transcripts of genes related to lipid metabolism and transportation ${ }^{53-58}$, are expressed in YSL during early development to facilitate the hydrolysis and transport of yolk lipids. When these genes are disrupted, embryos show reduced yolk consumption, delayed embryonic growth and failure in lipid absorption ${ }^{58,59}$.

The presence of GFP signal in YSL also suggested a role for LC-PUFA, the product of $f a d s 2$ promoter activities, in transportation of yolk lipids. Detailed analyses of lipid classes in zebrafish yolk imply active de novo synthesis of lipids, in which fatty acids are resynthesised into major lipid classes between 0-48 hpf stage, followed by packaging into very low-density lipoproteins for supply to whole embryo beyond $48 \mathrm{hpf}^{15,19}$. In Caenorhabditis elegans, transportation of yolk lipoprotein to oocytes was disrupted in mutants with PUFA deficiency, a malaise restored by feeding worms with n-6 PUFA ${ }^{60}$. These findings, viewed alongside our results showing localisation of fads 2 promoter activity at the YSL, provides the collective interpretation that LC-PUFA is required for transportation of yolk lipids during development.

In adult teleost, the liver is a major site for LC-PUFA biosynthesis, with both fads and elovl transcripts being reported in adult zebrafish liver and early larval stages ${ }^{18,21}$. Since the injected embryos only showed expression in the YSL, the fads 2 promoter fragment lacks the regulatory region responsible for directing expression of fads 2 in other organs such as liver, intestine or brain. It is plausible that the regulatory elements for the expression of these tissues are located in more distal regions or/and introns ${ }^{61,62}$. Multiple studies have shown that recapitulation of endogenous expression by a promoter in zebrafish embryos depends on the length of the promoter fragment ${ }^{62-64}$.

In conclusion, we successfully isolated a 1754 bp upstream region of the zebrafish fads 2 promoter and identified several response elements critical to drive the activation of transcription. We also showed the capacity of this fragment to direct GFP expression at the YSL region of $26 \mathrm{hpf}$ and older stages embryos. Since zebrafish relies on yolk as sole lipid source prior to onset of exogenous feeding capacity, it potentially provides a platform to further elucidate the role of $f a d s 2$ or LC-PUFA in transportation of yolk lipids across the YSL.

\section{Materials and Methods}

Cell line maintenance and subculture. The zebrafish liver cell line, ZFL (ATCC ${ }^{\circledR}$ CRL-2643 ${ }^{\mathrm{TM}}$ ) was purchased from the American Type Culture Collection (ATCC, USA) and maintained in the complete growth medium at $28^{\circ} \mathrm{C}$ following the protocol of ATCC. Routine subculture was performed once the ZFL cells have achieved 80 to $90 \%$ confluence in a $25 \mathrm{~cm}^{2}$ flask.

Zebrafish maintenance and embryo collection. Wild type zebrafish (AB line) were purchased from the Institute of Molecular and Cell Biology (IMCB, Singapore), and maintained in the ZebTEC Stand Alone System (Tecniplast, USA). The fish were kept on a $13: 11 \mathrm{~h}$ light/dark cycle at $28.5^{\circ} \mathrm{C}$, and fed till visual satiation twice daily with a combination of commercial micro pellet (Aquadene, Malaysia), frozen bloodworms and Artemia nauplii. Breeding and embryo collection were carried out according to Westerfield (2000) with adaptation ${ }^{65}$.

Animal ethics approval. Husbandry, handling and use of animals in this manuscript comply with the guidelines and requirements of the Animal Ethics Committee, Universiti Sains Malaysia and were approved by the same committee (PA/ACSC/002/2011).

Zebrafish fads2 promoter cloning and promoter-luciferase reporter plasmid construction. A promoter region of $1754 \mathrm{bp}$, corresponding to $-1735 /+19 \mathrm{bp}$ relative to the transcription start site of the zebrafish fads 2 gene was amplified with PCR utilising the $i$-Taq ${ }^{\text {TM }}$ Plus DNA Polymerase (iNtRON, Korea). DNA extracted from the ZFL cells was used as the template for the amplification, together with the forward primer F1735 containing the restriction site for KpnI, and a reverse primer R19 containing the restriction site for XhoI (Supplementary data 3 ). The reaction mixtures were subjected to a thermal cycling programme consisted of an 
initial denaturation step for $2 \mathrm{~min}$ at $94^{\circ} \mathrm{C}$, followed by 40 cycles of denaturation for $20 \mathrm{~s}$ at $94^{\circ} \mathrm{C}$, annealing for $20 \mathrm{~s}$ at $50^{\circ} \mathrm{C}$, and extension for $1 \mathrm{~min} / \mathrm{kb}$ of product length at $72{ }^{\circ} \mathrm{C}$, and a final extension step for $5 \mathrm{~min}$ at $72^{\circ} \mathrm{C}$. The resulted PCR products were gel purified, cloned into the pGL3-Basic luciferase reporter vector (Promega, USA) and sequenced. Similarly, for the $5^{\prime}$-deletion analysis, another five promoter-luciferase reporter plasmids containing the fads 2 promoter region with deletions of different lengths at the $5^{\prime}$-end were generated by using a series of forward primers (F1145, F586, F244, F161 or F67) and a common reverse primer (R19).

Zebrafish Srebp expression plasmid construction. Total RNA was extracted from the adult zebrafish liver using the TRIzol ${ }^{\circledR}$ Reagent (Invitrogen, Germany) according to the manufacturer's descriptions. First-strand cDNA was generated via reverse transcription PCR using the M-MLV Reverse Transcriptase (Promega, USA). PCR fragments corresponding to the N-terminal of zebrafish srebp-1 (nSrebp-1, 1-457 aa; NM_001105129.1) and srebp-2 (nSrebp-2, 1-464 aa; NM_001089466.1) were identified through alignment with the human SREBP homologues (Nakakuki et al., 2007), and amplified from the first-strand cDNA using forward primers containing the restriction site for KpnI and reverse primers containing a stop codon (TAG) for translation termination and the restriction site for XhoI (Supplementary data 4). PCR amplification was performed as abovementioned and the resulted PCR products were cloned into the CMV promoter-driven expression plasmid pcDNA3.1 (Invitrogen, Germany).

Transient transfection and dual luciferase reporter assay. Prior to the day of transient transfection, $2.5 \times 10^{4}$ ZFL cells per well were seeded in a 96-well clear bottom white walled plates (Thermo Scientific, USA) and incubated overnight for cell attachment. Transient transfection was carried out using transfection reagent Lipofectamine $^{\mathrm{TM}} 2000$ (Life Technologies, Germany) at a volume of $0.15 \mu$ l per $100 \mathrm{ng}$ DNA. For nSrebp overexpression assay, ZFL cells were co-transfected with fads2-1735 promoter-luciferase reporter plasmid (100 ng/well), one of the nSrebp expression plasmids (50 ng/well) and Renilla luciferase reference plasmid, pRL-SV40 (50 ng/ well). ZFL cells prepared for the $5^{\prime}$-deletion assay were co-transfected with one of the pGL3 promoter-luciferase reporter plasmids (equimolar to $100 \mathrm{ng}$ fads2-1735 plasmid/well), pcDNA3.1-nSrebp2 (50 ng/well) and pRL-SV40 (50 ng/well). Co-transfection for mutation analysis was performed with one of the mutated pGL3 promoter-luciferase reporter plasmids (100 ng/well), pcDNA3.1-nSrebp2 (50 ng/well) and pRL-SV40 (50 ng/ well). Lastly, nuclear extracts for EMSA (refer to section 2.7) were prepared from the ZFL cells seeded in a $25 \mathrm{~cm}^{2}$ flasks and transfected with pcDNA3.1-nSrebp $2(2 \mu \mathrm{g} / \mathrm{ml})$. Briefly, plasmid DNA-Lipofectamine ${ }^{\mathrm{TM}} 2000$ complexes in $50 \mu \mathrm{l} \mathrm{Opti-MEM} \mathrm{were} \mathrm{equally} \mathrm{distributed} \mathrm{to} \mathrm{each} \mathrm{well} \mathrm{and} \mathrm{the} \mathrm{ZFL} \mathrm{cells} \mathrm{were} \mathrm{incubated} \mathrm{for} 4.5 \mathrm{~h}$ at $28.5^{\circ} \mathrm{C}$. The plasmid DNA-Lipofectamine ${ }^{\mathrm{TM}} 2000$ complexes contained-culture medium was then replaced with fresh culture medium and the transfected ZFL cells were further incubated for $24 \mathrm{~h}$ before being proceeded to subsequent assay. The ZFL cells were harvested in $20 \mu \mathrm{l} /$ well Passive Lysis Buffer and luciferase assay was carried out using the Dual-Glo ${ }^{\circledR}$ Luciferase Assay System (Promega, USA) according to the manufacturer's protocols. All the luminescence measurements were performed in the GloMax ${ }^{\circledR}$ Multi + Detection System (Promega, USA). Firefly luminescence reading for each sample was first normalised to Renilla luminescence reading as the internal control of transfection efficiency, before it was further normalised to the control cells transfected with pGL3-Basic plasmid for the comparison of promoter activities.

Site-directed mutagenesis. Multiple base substitution mutations at the SRE site, proximal and distal CCAAT box sites were created in the fads2-244 plasmid employing the Muta-Direct ${ }^{\mathrm{TM}}$ Site-Directed Mutagenesis Kit (iNtRON, Korea) following the manufacturer's instructions. Complementary mutagenic primers (Supplementary data 5) used in the mutagenesis were designed using web-based programme PrimerX (http:// www.bioinformatics.org/primerx/). Multiple site mutations were carried out one after another by using previously constructed mutant plasmids as templates.

Electrophoretic mobility shift assay. Prior to electrophoretic mobility shift assay (EMSA), $3^{\prime}$-end of each complementary strand of the oligonucleotides (Supplementary data 6) were labelled with biotin using the Biotin $3^{\prime}$ End DNA Labelling Kit (Thermo Scientific, USA) following descriptions of the manufacturer. Subsequently, biotin-labelled double-stranded oligonucleotides $(10 \mu \mathrm{M})$ were generated by mixing equal amounts of each complementary strand of the resulted oligonucleotides at least $1 \mathrm{~h}$ prior to EMSA. For non-labelled competition double-stranded oligonucleotides, equimolar of each complementary strand of oligonucleotides $(10 \mu \mathrm{M})$ were added into $1 \mathrm{X}$ annealing buffer containing $10 \mathrm{mM}$ Tris ( $\mathrm{pH} 7.5$ ), $1 \mathrm{mM}$ EDTA and $50 \mathrm{mM} \mathrm{NaCl}$, and the mixture was heated in the Thermomix (Eppendorf, Germany) for $5 \mathrm{~min}$ at $95^{\circ} \mathrm{C}$ before gradually cooling down to room temperature over a period of approximately $1 \mathrm{~h}$. Nuclear extract was prepared from the transfected ZFL cells using the NE-PER ${ }^{\circledR}$ Nuclear and Cytoplasmic Extraction Reagents (Thermo Scientific, USA) according to the manufacturer's instructions. For EMSA, the LightShift ${ }^{\circledR}$ Chemiluminescent EMSA Kit (Thermo Scientific, USA) was used and each binding reaction containing $1 \mathrm{X}$ binding buffer, $1 \mu \mathrm{g}$ Poly (dI.dC), 5\% (v/v) glycerol, $0.05 \%$ (v/v) NP-40, $50 \mathrm{mM} \mathrm{KCl,} 5 \mathrm{mM} \mathrm{MgCl}$ 2, $20 \mathrm{mM}$ EDTA, $5 \mu \mathrm{l}$ nuclear extract and $100 \mathrm{fmol}$ biotin-labelled oligonucleotides in a total volume of $20 \mu \mathrm{ddd} 2 \mathrm{O}$ was prepared. For the negative control binding reaction, nuclear extract was excluded, and 100-fold molar excess of non-labelled oligonucleotides was included to the competition binding reaction. The DNA-protein binding complexes were then incubated at room temperature for $30 \mathrm{~min}$ before size-fractioning on a $5 \%(\mathrm{v} / \mathrm{v})$ non-denaturing polyacrylamide gel at $4{ }^{\circ} \mathrm{C}$ in the Mini-PROTEAN II Slab Electrophoresis Cell (Bio-Rad, USA). Finally, biotin-labelled DNA was detected with the aid of Chemiluminescent Nucleic Acid Detection Module (Thermo Scientific, USA) and luminescent signal was captured using the CCD camera (Bio-Rad, USA). 
GFP promoter-reporter plasmid construction. DNA fragments from the fads $2-244$ and -1735 promoter-luciferase reporter plasmids were PCR amplified using both forward and reverse primers containing the restriction site for XhoI and BamHI (Supplementary data 3), respectively. The resulted PCR products were gel-purified and cloned into the pZsGreen1-1 GFP promoter-reporter plasmid.

Transient expression of GFP promoter-reporter plasmid in zebrafish embryo. In vivo promoter activity of both promoter fragments was determined by microinjection of GFP promoter-reporter plasmids into zebrafish embryos, respectively. Prior to microinjection, GFP promoter-reporter plasmids were linearised with the restriction enzymes XhoI and NotI. Each pZsGreen1-1 GFP promoter-reporter construct was adjusted to $10 \mathrm{ng} / \mu \mathrm{l}$ with $\mathrm{dH}_{2} \mathrm{O}$ containing $1 \mathrm{X}$ Danieau's buffer and $0.2 \%$ phenol red. Approximately, $4.6 \mathrm{nl}$ of the DNA solution was delivered into embryos at early one-cell stage by using the Nanoliter 2000 Microinjector (World Precision Instrument, USA). The injected embryos were incubated in E3 medium at $28.5^{\circ} \mathrm{C}$. The embryos were periodically monitored under the MVX10 Fluorescence Macro Zoom Microscope equipped with ColorView III Soft Imaging System (Olympus, Japan) until $120 \mathrm{hpf}$. For cryosectioning, zebrafish young larvae were fixed with $4 \%$ paraformaldehyde, treated and sectioned at a thickness of $20 \mu \mathrm{m}$ using the Leica CM1850 UV Cryostat (Leica Biosystems, Germany).

\section{References}

1. Leaver, M. J. et al. Towards Fish Lipid Nutrigenomics: Current State and Prospects for Fin-Fish Aquaculture. Rev. Fish. Sci. 16, 73-94, https://doi.org/10.1080/10641260802325278 (2008).

2. Tocher, D. R. Omega-3 long-chain polyunsaturated fatty acids and aquaculture in perspective. Aquaculture 449, 94-107, https://doi. org/10.1016/j.aquaculture.2015.01.010(2015).

3. Marquardt, A., Stohr, H., White, K. \& Weber, B. H. cDNA cloning, genomic structure, and chromosomal localization of three members of the human fatty acid desaturase family. Genomics 66, 175-183, https://doi.org/10.1006/geno.2000.6196 (2000).

4. Castro, L. F. C. et al. Functional desaturase Fads1 $\Delta 5$ and Fads $2 \Delta 6$ orthologues evolved before the origin of jawed vertebrates. PLoS ONE 7, e31950, https://doi.org/10.1371/journal.pone.0031950 (2012).

5. Hastings, N. et al. A vertebrate fatty acid desaturase with $\Delta 5$ and $\Delta 6$ activities. Proc. Natl. Acad. Sci. USA 98, 14304-14309, https:// doi.org/10.1073/pnas.251516598 (2001)

6. Li, Y. et al. Vertebrate fatty acyl desaturase with Delta4 activity. Proc Natl Acad Sci USA 107, 16840-16845, https://doi.org/10.1073/ pnas.1008429107 (2010).

7. Monroig, Ó., Tocher, D. R., Hontoria, F. \& Navarro, J. C. Functional characterisation of a Fads 2 fatty acyl desaturase with $\Delta 6 / \Delta 8$ activity and an Elovl5 with C16, C18 and C20 elongase activity in the anadromous teleost meagre (Argyrosomus regius). Aquaculture 412-413, 14-22, https://doi.org/10.1016/j.aquaculture.2013.06.032 (2013).

8. Zheng, X. et al. Characterization and comparison of fatty acyl $\Delta 6$ desaturase cDNAs from freshwater and marine teleost fish species. Comp. Biochem. Physiol. B 139, 269-279 (2004).

9. Morais, S., Castanheira, F., Martinez-Rubio, L., Conceição, L. E. C. \& Tocher, D. R. Long chain polyunsaturated fatty acid synthesis in a marine vertebrate: Ontogenetic and nutritional regulation of a fatty acyl desaturase with $\triangle 4$ activity. BBA-Mol. Cell Biol. Lipids 1821, 660-671, https://doi.org/10.1016/j.bbalip.2011.12.011 (2012).

10. Zheng, X., Leaver, M. J. \& Tocher, D. R. Long-chain polyunsaturated fatty acid synthesis in fish: Comparative analysis of Atlantic salmon (Salmo salar L.) and Atlantic cod (Gadus morhua L.) Delta6 fatty acyl desaturase gene promoters. Comp Biochem Physiol B Biochem Mol Biol 154, 255-263, https://doi.org/10.1016/j.cbpb.2009.06.010 (2009).

11. Geay, F. et al. Characteristics of fads2 gene expression and putative promoter in European sea bass (Dicentrarchus labrax): comparison with salmonid species and analysis of CpG methylation. Mar. Genomics 5, 7-13, https://doi.org/10.1016/j. margen.2011.08.003 (2012).

12. Xu, H. G. et al. Regulation of Tissue LC-PUFA Contents, D6 Fatty Acyl Desaturase (FADS2) Gene Expression and the Methylation of the Putative FADS2 Gene Promoter by Different Dietary Fatty Acid Profiles in Japanese Seabass (Lateolabrax japonicus). Plos One 9, https://doi.org/10.1371/journal.pone.0087726 (2014).

13. Dong, X. et al. Regulation of FADS2 transcription by SREBP-1 and PPAR-alpha influences LC-PUFA biosynthesis in fish. Sci. Rep. 7, 40024, https://doi.org/10.1038/srep40024 (2017).

14. Dong, Y. et al. Cloning and characterization of 6/5 fatty acyl desaturase (Fad) gene promoter in the marine teleost Siganus canaliculatus. Gene, https://doi.org/10.1016/j.gene.2018.01.031 (2018).

15. Miyares, R. L., de Rezende, V. B. \& Farber, S. A. Zebrafish yolk lipid processing: a tractable tool for the study of vertebrate lipid transport and metabolism. Dis Model Mech 7, 915-927, https://doi.org/10.1242/dmm.015800 (2014).

16. Fraher, D. et al. Zebrafish embryonic lipidomic analysis reveals that the yolk cell is metabolically active in processing lipid. Cell Rep 14, 1317-1329, https://doi.org/10.1016/j.celrep.2016.01.016 (2016).

17. Holtta-Vuori, M. et al. Zebrafish: gaining popularity in lipid research. Biochem J 429, 235-242, https://doi.org/10.1042/BJ20100293 (2010).

18. Monroig, O., Rotllant, J., Sanchez, E., Cerda-Reverter, J. M. \& Tocher, D. R. Expression of long-chain polyunsaturated fatty acid (LCPUFA) biosynthesis genes during zebrafish Danio rerio early embryogenesis. BBA-Mol. Cell Biol. Lipids 1791, 1093-1101 (2009).

19. Pirro, V. et al. Lipid dynamics in zebrafish embryonic development observed by DESI-MS imaging and nanoelectrospray-MS. Mol Biosyst 12, 2069-2079, https://doi.org/10.1039/c6mb00168h (2016).

20. Agaba, M., Tocher, D. R., Dickson, C. A., Dick, J. R. \& Teale, A. J. Zebrafish cDNA encoding multifunctional fatty acid elongase involved in production of eicosapentaenoic (20:5n-3) and docosahexaenoic (22:6n-3) acids. Mar. Biotechnol. 6, 251-261, https://doi. org/10.1007/s10126-003-0029-1 (2004).

21. Tan, S.-H., Chung, H.-H. \& Shu-Chien, A. C. Distinct developmental expression of two elongase family members in zebrafish. Biochem. Bioph. Res. Co. 393, 397-403, https://doi.org/10.1016/j.bbrc.2010.01.130 (2010).

22. Monroig, O. et al. Expression and role of Elovl4 elongases in biosynthesis of very long-chain fatty acids during zebrafish Danio rerio early embryonic development. BBA-Mol. Cell Biol. Lipids 1801, 1145-1154 (2010).

23. Bhandari, S. et al. The fatty acid chain elongase, Elovl1, is required for kidney and swim bladder development during zebrafish embryogenesis. Organogenesis 12, 78-93, https://doi.org/10.1080/15476278.2016.1172164 (2016).

24. Nara, T. Y., He, W. S., Tang, C. G., Clarke, S. D. \& Nakamura, M. T. The E-box like sterol regulatory element mediates the suppression of human Delta-6 desaturase gene by highly unsaturated fatty acids. Biochem. Bioph. Res. Co. 296, 111-117, https://doi.org/10.1016/ S0006-291X(02)00851-3 (2002)

25. Jaya-Ram, A., Kuah, M. K., Lim, P. S., Kolkovski, S. \& Shu-Chien, A. C. Influence of dietary HUFA levels on reproductive performance, tissue fatty acid profile and desaturase and elongase mRNAs expression in female zebrafish Danio rerio. Aquaculture 277, 275-281 (2008).

26. Briggs, M. R., Yokoyama, C., Wang, X., Brown, M. S. \& Goldstein, J. L. Nuclear protein that binds sterol regulatory element of low density lipoprotein receptor promoter. I. Identification of the protein and delineation of its target nucleotide sequence. J Biol Chem 268, 14490-14496 (1993). 
27. Minghetti, M., Leaver, M. J. \& Tocher, D. R. Transcriptional control mechanisms of genes of lipid and fatty acid metabolism in the Atlantic salmon (Salmo salar L.) established cell line, SHK-1. Biochim Biophys Acta 1811, 194-202, https://doi.org/10.1016/j. bbalip.2010.12.008 (2011).

28. Passeri, M. J., Cinaroglu, A., Gao, C. \& Sadler, K. C. Hepatic steatosis in response to acute alcohol exposure in zebrafish requires sterol regulatory element binding protein activation. Hepatology 49, 443-452, https://doi.org/10.1002/hep.22667 (2009).

29. Carmona-Antonanzas, G., Tocher, D. R., Martinez-Rubio, L. \& Leaver, M. J. Conservation of lipid metabolic gene transcriptional regulatory networks in fish and mammals. Gene 534, 1-9, https://doi.org/10.1016/j.gene.2013.10.040 (2014).

30. Zhang, Q. et al. Cloning and Characterization of Lxr and Srebp1, and Their Potential Roles in Regulation of LC-PUFA Biosynthesis in Rabbitfish Siganus canaliculatus. Lipids 51, 1051-1063, https://doi.org/10.1007/s11745-016-4176-3 (2016).

31. Leaver, M. J. et al. Functional genomics reveals increases in cholesterol biosynthetic genes and highly unsaturated fatty acid biosynthesis after dietary substitution of fish oil with vegetable oils in Atlantic salmon (Salmo salar). Bmc Genomics 9, https://doi. org/10.1186/1471-2164-9-299 (2008).

32. Dong, X. J. et al. Cloning and characterization of SREBP-1 and PPAR-alpha in Japanese seabass Lateolabrax japonicus, and their gene expressions in response to different dietary fatty acid profiles. Comp Biochem Phys B 180, 48-56, https://doi.org/10.1016/j. cbpb.2014.10.001 (2015).

33. Tang, C., Cho, H. P., Nakamura, M. T. \& Clarke, S. D. Regulation of human delta- 6 desaturase gene transcription: identification of a functional direct repeat-1 element. J Lipid Res 44, 686-695, https://doi.org/10.1194/jlr.M200195-JLR200 (2003).

34. Kumadaki, S. et al. Mouse Elovl-6 promoter is an SREBP target. Biochem Biophys Res Commun 368, 261-266, https://doi. org/10.1016/j.bbrc.2008.01.075 (2008).

35. Mantovani, R. The molecular biology of the CCAAT-binding factor NF-Y. Gene 239, 15-27 (1999)

36. Dolfini, D. \& Gatta, R. \& Mantovani, R. NF-Y and the transcriptional activation of CCAAT promoters. Crit Rev Biochem Mol Biol 47, 29-49, https://doi.org/10.3109/10409238.2011.628970 (2012).

37. Ericsson, J., Jackson, S. M., Kim, J. B., Spiegelman, B. M. \& Edwards, P. A. Identification of glycerol-3-phosphate acyltransferase as an adipocyte determination and differentiation factor 1- and sterol regulatory element-binding protein-responsive gene. J Biol Chem 272, 7298-7305 (1997).

38. Jackson, S. M., Ericsson, J., Osborne, T. F. \& Edwards, P. A. NF-Y has a novel role in sterol-dependent transcription of two cholesterogenic genes. J Biol Chem 270, 21445-21448 (1995).

39. Tabor, D. E., Kim, J. B., Spiegelman, B. M. \& Edwards, P. A. Identification of conserved cis-elements and transcription factors required for sterol-regulated transcription of stearoyl-CoA desaturase 1 and 2. J Biol Chem 274, 20603-20610 (1999).

40. Yieh, L., Sanchez, H. B. \& Osborne, T. F. Domains of transcription factor Sp1 required for synergistic activation with sterol regulatory element binding protein 1 of low density lipoprotein receptor promoter. Proc Natl Acad Sci USA 92, 6102-6106 (1995).

41. Teran-Garcia, M. et al. Polyunsaturated fatty acid suppression of fatty acid synthase (FASN): evidence for dietary modulation of NF-Y binding to the Fasn promoter by SREBP-1c. Biochem J 402, 591-600, https://doi.org/10.1042/BJ20061722 (2007).

42. Shimano, H. Sterol regulatory element-binding proteins (SREBPs): transcriptional regulators of lipid synthetic genes. Progress in Lipid Research 40, 439-452 (2001).

43. Amemiya-Kudo, M. et al. Promoter analysis of the mouse sterol regulatory element-binding protein-1c gene. J. Biol. Chem 275, 31078-31085, https://doi.org/10.1074/jbc.M005353200 (2000).

44. Magana, M. M. \& Osborne, T. F. Two tandem binding sites for sterol regulatory element binding proteins are required for sterol regulation of fatty-acid synthase promoter. J Biol Chem 271, 32689-32694 (1996).

45. Magana, M. M., Koo, S. H., Towle, H. C. \& Osborne, T. F. Different sterol regulatory element-binding protein-1 isoforms utilize distinct co-regulatory factors to activate the promoter for fatty acid synthase. J Biol Chem 275, 4726-4733 (2000).

46. Wiegand, M. D. Utilization of yolk fatty acids by goldfish embryos and larvae. Fish Physiol Biochem 15, 21-27, https://doi. org/10.1007/BF01874834 (1996).

47. Wiegand, M. D. C. accumulation and utilization of yolk lipids in teleost fish. Rev Fish Biol Fisher 6, 259-286, https://doi.org/10.1007/ Bf00122583 (1996).

48. Kimmel, C. B. \& Law, R. D. Cell lineage of zebrafish blastomeres. II. Formation of the yolk syncytial layer. Dev Biol 108, 86-93 (1985).

49. Sakaguchi, T., Mizuno, T. \& Takeda, H. Formation and patterning roles of the yolk syncytial layer. Results Probl Cell Differ 40, 1-14 (2002).

50. Kondakova, E. A. \& Efremov, V. I. Morphofunctional transformations of the yolk syncytial layer during zebrafish development. J Morphol 275, 206-216, https://doi.org/10.1002/jmor.20209 (2014).

51. Carvalho, L. \& Heisenberg, C. P. The yolk syncytial layer in early zebrafish development. Trends Cell Biol 20, 586-592, https://doi. org/10.1016/j.tcb.2010.06.009 (2010).

52. Lepage, S. E. \& Bruce, A. E. Zebrafish epiboly: mechanics and mechanisms. Int J Dev Biol 54, 1213-1228, https://doi.org/10.1387/ ijdb.093028sl (2010).

53. Babin, P. J. et al. Both apolipoprotein $\mathrm{E}$ and A-I genes are present in a nonmammalian vertebrate and are highly expressed during embryonic development. Proc Natl Acad Sci USA 94, 8622-8627 (1997).

54. Poupard, G. et al. Apolipoprotein E gene expression correlates with endogenous lipid nutrition and yolk syncytial layer lipoprotein synthesis during fish development. Cell Tissue Res 300, 251-261 (2000).

55. Mudumana, S. P., Wan, H., Singh, M., Korzh, V. \& Gong, Z. Expression analyses of zebrafish transferrin, ifabp, and elastaseB mRNAs as differentiation markers for the three major endodermal organs: liver, intestine, and exocrine pancreas. Dev Dyn 230, 165-173, https://doi.org/10.1002/dvdy.20032 (2004).

56. Marza, E. et al. Developmental expression and nutritional regulation of a zebrafish gene homologous to mammalian microsomal triglyceride transfer protein large subunit. Dev. Dyn. 232, 506-518, https://doi.org/10.1002/dvdy.20251 (2005).

57. Wang, R., Li, Z., Wang, Y. \& Gui, J. F. An Apo-14 promoter-driven transgenic zebrafish that marks liver organogenesis. PLoS One 6, e22555, https://doi.org/10.1371/journal.pone.0022555 (2011).

58. Chang, N. Y. et al. Sterol O-Acyltransferase 2 Contributes to the Yolk Cholesterol Trafficking during Zebrafish Embryogenesis. PLoS One 11, e0167644, https://doi.org/10.1371/journal.pone.0167644 (2016).

59. Schlegel, A. \& Stainier, D. Y. Microsomal triglyceride transfer protein is required for yolk lipid utilization and absorption of dietary lipids in zebrafish larvae. Biochemistry 45, 15179-15187, https://doi.org/10.1021/bi0619268 (2006).

60. Chen, W. W. et al. Specific polyunsaturated fatty acids modulate lipid delivery and oocyte development in C. elegans revealed by molecular-selective label-free imaging. Sci. Rep. 6, 32021, https://doi.org/10.1038/srep32021 (2016).

61. Muller, F. et al. Intronic enhancers control expression of zebrafish sonic hedgehog in floor plate and notochord. Development 126, 2103-2116 (1999).

62. Shentu, H. et al. Proximal upstream region of zebrafish bone morphogenetic protein 4 promoter directs heart expression of green fluorescent protein. Genesis 37, 103-112, https://doi.org/10.1002/gene.10240 (2003).

63. Her, G. M., Yeh, Y. H. \& Wu, J. L. Functional conserved elements mediate intestinal-type fatty acid binding protein (I-FABP) expression in the gut epithelia of zebrafish larvae. Dev Dyn 230, 734-742, https://doi.org/10.1002/dvdy.20081 (2004).

64. Burns, F. R. et al. Analysis of the zebrafish sox $9 \mathrm{~b}$ promoter: Identification of elements that recapitulate organ-specific expression of sox9b. Gene 578, 281-289, https://doi.org/10.1016/j.gene.2015.12.041 (2016).

65. Westerfield, M. The Zebrafish Book: A Guide for the Laboratory Use of the Zebrafish (Danio rerio). (University of Oregon, 1994). 


\section{Acknowledgements}

We thank the Malaysian Ministry of Higher Education for funding this work (ERGS, 203/PBIOLOGI/6730050). A Universiti Sains Malaysia Post-Doctoral fellowship to MK Kuah is also acknowledged.

\section{Author Contributions}

Shu-Chien A.C., M.K. Kuah and S.S. Tay designed the studies. S.S. Tay and M.K. Kuah conducted the research. All authors analysed the data, wrote, read and approved the final manuscript.

\section{Additional Information}

Supplementary information accompanies this paper at https://doi.org/10.1038/s41598-018-22157-4.

Competing Interests: The authors declare no competing interests.

Publisher's note: Springer Nature remains neutral with regard to jurisdictional claims in published maps and institutional affiliations.

(c) (i) Open Access This article is licensed under a Creative Commons Attribution 4.0 International License, which permits use, sharing, adaptation, distribution and reproduction in any medium or format, as long as you give appropriate credit to the original author(s) and the source, provide a link to the Creative Commons license, and indicate if changes were made. The images or other third party material in this article are included in the article's Creative Commons license, unless indicated otherwise in a credit line to the material. If material is not included in the article's Creative Commons license and your intended use is not permitted by statutory regulation or exceeds the permitted use, you will need to obtain permission directly from the copyright holder. To view a copy of this license, visit http://creativecommons.org/licenses/by/4.0/.

(C) The Author(s) 2018 